\title{
Dimers of Nostocarboline with Potent Antibacterial Activity
}

\author{
Hans H. Locher ${ }^{a} \quad$ Daniel Ritz $^{a}$ Philippe Pfaffa ${ }^{a}$ Mika Gaertner ${ }^{a}$ \\ Andreja Knezevic $^{a} \quad$ Daniela Sabato $^{a}$ Susanne Schroeder ${ }^{a}$ Damien Barbaras $^{b}$ \\ Karl Gademann ${ }^{c}$ \\ ${ }^{a}$ Actelion Pharmaceuticals Ltd., Allschwil, ${ }^{b}$ Laboratorium für Organische Chemie, Swiss Federal Institute of \\ Technology (ETH), Zürich, and 'Department of Chemistry, University of Basel, Basel, Switzerland
}

\section{Key Words}

Cationic antibacterials $\cdot$ Chlorhexidine $\cdot$ Mode of action

\begin{abstract}
Objectives: In this study, the in vitro antimicrobial activity and spectrum of new dimeric compounds derived from the cyanobacterial alkaloid nostocarboline were investigated. The mechanism of action and selectivity to bacteria were studied and compared to the cationic antiseptic chlorhexidine. Methods: Minimal inhibitory concentrations were determined against clinical isolates and against a panel of microbial reference strains using the CLSI microdilution method. Bacterial membrane damage was addressed by measuring ATP leakage and the mode of action was investigated in Escherichia coli reporter strains. Selectivity was tested by a cytotoxicity assay using MTS. Results: The antimicrobial potency of dimers varied with length of the hydrophobic linker. The most potent compounds, NCD9 and NCD10, had a C10 and C12 linker, respectively, and showed strong activity against Gram-positive bacteria, notably methicillinresistant Staphylococcus aureus strains. Similar to chlorhexidine, these compounds showed a rapid concentrationdependent bactericidal effect, which correlated with membrane damage as indicated by ATP leakage. NCD9, in contrast to NCD10 and chlorhexidine, lacked activity against yeast
\end{abstract}

strains and showed low cytotoxicity in $\mathrm{CHO}$ cells indicating a high degree of selectivity. In E. coli reporter strains, NCD9 induced the DegP response pathway as well as the SOS response, suggesting interaction with both the cell envelope and DNA metabolism. Conclusions: The results presented in this report indicate the potential of this new class of cationic antimicrobial compounds for the design of potent and selective antibacterials with low cytotoxicity.

Copyright $\odot 2010$ S. Karger AG, Basel

\section{Introduction}

The problem of microbial drug resistance has reached a global dimension and an alarming magnitude. Epidemic antibiotic resistance has been reported in numerous pathogens, including the global pandemic of methicillinresistant Staphylococcus aureus (MRSA) infection, and epidemic increases of multidrug-resistant and increasingly pan-resistant strains of Gram-negative bacteria, such as Pseudomonas aeruginosa and Acinetobacter baumannii [1-3]. The systemic or topical treatment of the increasing number of infections caused by resistant or multiresistant bacteria demands new antibiotics, preferably from new structural classes and with new mechanisms of action. However, the difficulty of finding and developing

\section{KARGER}

Fax +4161306 1234 E-Mail karger@karger.ch www.karger.com (c) 2010 S. Karger AG, Basel 0009-3157/10/0564-0318\$26.00/0

Accessible online at:

www.karger.com/che
Hans H. Locher

Actelion Pharmaceutical Ltd.

Gewerbestrasse 16

$\mathrm{CH}-4123$ Allschwil (Switzerland)

Tel. +41 6156580 69, Fax +41 6156580 90, E-Mail hans.locher@actelion.com 
Table 1. MICs (mg/l) by microdilution method against a panel of bacterial and yeast strains

\begin{tabular}{|c|c|c|c|c|c|c|c|c|c|c|c|c|c|}
\hline & \multicolumn{13}{|c|}{ Compounds } \\
\hline & NCD1 & NCD2 & NCD3 & NCD4 & NCD5 & NCD6 & NCD7 & NCD8 & NCD9 & NCD10 & $\mathrm{NC}$ & CIP & CHL \\
\hline S. aureus ATCC 29213 & 16 & 4 & 32 & 8 & $>32$ & 32 & 8 & 2 & 0.25 & 0.25 & $>32$ & 0.25 & 0.25 \\
\hline S. aureus A-798 (MRSA) & 16 & 8 & $>32$ & 8 & 32 & 8 & 16 & 8 & 0.5 & 0.5 & $>32$ & $>32$ & 0.25 \\
\hline E. faecium A-949 (VRE) & 32 & 8 & 32 & 8 & 32 & 32 & 32 & 16 & 2 & 1 & $>32$ & $>32$ & 2 \\
\hline S. pneumoniae ATCC 49619 & $>32$ & $>32$ & $>32$ & 32 & $>32$ & $>32$ & $>32$ & 32 & 4 & 4 & $>32$ & 1 & 4 \\
\hline H. influenzae A-921 & $>32$ & $>32$ & $>32$ & $>32$ & $>32$ & $>32$ & $>32$ & $>32$ & 8 & 32 & $>32$ & $\leq 0.03$ & 0.25 \\
\hline E. coli ATCC 25922 & 32 & $>32$ & $>32$ & $>32$ & $>32$ & $>32$ & 32 & 8 & 8 & 8 & $>32$ & $\leq 0.03$ & 0.125 \\
\hline E. coli Ec2085 & 2 & 8 & 8 & $>32$ & $>32$ & 1 & 1 & 0.5 & 0.5 & 1 & $>32$ & 0.004 & 0.125 \\
\hline A. baumannii T-6474 & 32 & 32 & $>32$ & $>32$ & $>32$ & $>32$ & $>32$ & 32 & 16 & 8 & $>32$ & $>32$ & 16 \\
\hline P. aeruginosa ATCC 27853 & 32 & $>32$ & $>32$ & $>32$ & $>32$ & $>32$ & $>32$ & $>32$ & 32 & $>32$ & $>32$ & 0.5 & 4 \\
\hline S. cerevisiae A-136 & $>32$ & $>32$ & $>32$ & $>32$ & $>32$ & $>32$ & $>32$ & $>32$ & $>32$ & 1 & $>32$ & $>32$ & 4 \\
\hline C. albicans T-3419 & $>32$ & 32 & $>32$ & $>32$ & $>32$ & $>32$ & $>32$ & $>32$ & $>32$ & 0.5 & $>32$ & $>32$ & 4 \\
\hline
\end{tabular}

$\mathrm{VRE}=$ Vancomycin-resistant Enterococcus $; \mathrm{NC}=$ nostocarboline; CIP = ciprofloxacin; $\mathrm{CHL}=$ chlorhexidine.

new antibacterial agents is underlined by the fact that only two new chemical classes were brought into medical use over the past 35 years: the oxazolidinones (with linezolid as the only representative so far) and daptomycin [4].

Natural products have historically been a rich source of antimicrobial compounds and roughly $50 \%$ of all agents on the market addressing infectious diseases are natural products or derivatives thereof [5]. Cyanobacteria have been shown to be a prolific source of bioactive compounds [6-8]. The cyanobacterial metabolite nostocarboline has potent algicidal activity, while antibacterial activity is weak or absent [7-9]. This secondary metabolite, originally isolated from the freshwater cyanobacterium Nostoc 78-12A [9], has been demonstrated to inhibit the growth of other algae [7], a feature (alloleopathy) that is commonly found in cyanobacterial metabolites [8]. Recently, it was demonstrated that nostocarboline is active against the parasite Plasmodium falciparum and homodimers of nostocarboline with potent antiplasmodial activity were synthesized $[10,11]$. The mode of action of nostocarboline against Plasmodium was speculated to be correlated to its algicidal activity, which is unclear at present. Dimerization of cationic natural products often leads to enhanced biological activity and the resulting bisbicationic compounds are clinically used as antimicrobial agents [10]. Here, we report on the antibacterial activity and spectrum of a series of nostocarboline dimers as well as on investigations on the mode of action and selectivity of these compounds. Since dimerization of nostocarboline leads to bisbicationic compounds, we chose the mem- brane-acting bisbiguanide antiseptic chlorhexidine as a comparator. In addition, ciprofloxacin was used as a reference antibiotic and as an example of an antibacterial with a distinct intracellular target.

\section{Material and Methods}

\section{Antibacterial Compounds}

The synthesis of nostocarboline and its homodimers has been reported elsewhere $[10,11]$.

Ciprofloxacin and chlorhexidine were obtained from SigmaAldrich.

\section{Microbial Strains}

Strains used in this study were from the strain collection of Actelion Pharmaceuticals Ltd. Most of the clinical isolates were collected between 2001 and 2006 from various European and US hospitals. Reference strains were obtained from the American Type Culture Collection (ATCC). All strains were stored at $-80^{\circ} \mathrm{C}$ in $20 \%$ glycerol cultures. A primary panel of quality control strains from the ATCC as well as selected clinical isolates were used (table 1). Selected compounds were also tested against a panel of 20 MRSA clinical isolates. Genetically modified Escherichia coli strains were constructed in-house and used in reporter assays (see below). The stress response due to either DNA damage or damage to the cell envelope was assayed in strains derived from MG1655 (CGSC 7740). The luxCDABE operon of Photorhabdus luminescens [12] was linked to the trimethoprim resistance marker $d f r A 1$ from ColE1::Tn7 (DSMZ 3872) and then integrated in single copy in the chromosome using standard genetic procedures at the lac locus generating a $\triangle l a c Z:$ :(luxCDABE-dfrA1) strain. The sulA or $\operatorname{deg} P$ promoter region were PCR-amplified from the chromosome and fused by overlapping PCR to the gentamicin resistance cassette aph $\left(2^{\prime}\right)$-aac (6') from Enterococcus faecalis (ATCC 51299). Finally, the promoter region upstream of $l u x C$ was re- 
placed with either the sulA or $\operatorname{deg} P$ promoter following the procedure by Datsenko and Wanner [13]. The reporter strains were also made more susceptible to the effect of small molecules by introducing tolC [14] or $r f a C$ disruptions (Actelion strain collection) using generalized P1 transduction [15] resulting in strains Ec2376 [ $\Delta$ tolC $\Delta$ rfaC $\Delta$ lacZ::(sulA'-'luxCDABE)] and Ec2297 [ $\Delta$ tolC $\left.\Delta l a c Z::\left(\operatorname{deg} P^{\prime}-' l u x C D A B E\right)\right]$. An isogenic $\Delta$ tolC $\Delta r f a C$ mutant (Ec2085) was used for the determination of minimal inhibitory concentrations (MICs).

\section{Determination of MICs}

Stock solutions of compounds were made in DMSO $(5 \mathrm{mg} / \mathrm{ml})$. MICs were determined by a broth microdilution assay following the guidelines of the Clinical Laboratory Standards Institute (CLSI, formerly NCCLS) [16]. Cation-adjusted Mueller-Hinton II broth (CA-MHB) was used as the basic test medium (Becton Dickinson and Co., Sparks, Md., USA), which was supplemented as appropriate for fastidious organisms [16].

Time-Kill Assays

Exponential-phase S. aureus ATCC 29213 was diluted in fresh CA-MHB at approximately $10^{7}$ colony-forming units (cfu) per $\mathrm{ml}$. After $1 \mathrm{~h}$ of incubation with shaking at $37^{\circ} \mathrm{C}$, compounds were added at a concentration matching $4 \times$ and $16 \times$ the MIC. After further incubation for 1 and $4 \mathrm{~h}$, viable bacterial counts were determined by plating appropriate dilutions on Mueller-Hinton agar plates (Becton Dickinson and Co., Sparks, Md., USA), followed by determination of the cfu after $24 \mathrm{~h}$ incubation at $37^{\circ} \mathrm{C}$.

\section{ATP Release Assay}

Leakage of cellular ATP was measured to investigate the effect of compounds on membrane integrity [17]. Briefly, mid-exponential-phase $S$. aureus cells were centrifuged and resuspended in PBS containing antibacterial compounds. After incubation for 20 min at $37^{\circ} \mathrm{C}$ with shaking, suspensions were centrifuged again and the ATP concentration in the supernatant was determined using the BacTiter-Glo microbial cell viability assay kit (Promega, Madison, Wisc., USA). Untreated cultures served as the negative control while $0.1 \%$ (w/w) SDS was used as the positive (membranolytic) control.

\section{Stress Response Assays}

For the stress response assays, plates (Costar 3882, Corning, Mass., USA) were prepared as described for MIC assays, except that $10^{8}$ cells $/ \mathrm{ml}$ were added to the compounds. Luminescence was read after $2 \mathrm{~h}$ of incubation at $30^{\circ} \mathrm{C}$ with the compounds and normalized to the cells present in the wells (nRLU). The induction of the stress response was expressed as the ratio of nRLU measured with compounds and nRLU with $1 \%$ DMSO.

\section{Cytotoxicity}

Toxicity to eukaryotic cells was measured by using CHO-K1 cells and an MTS [3-(4,5-dimethylthiazol-2-yl)-5-(3-carboxymethoxyphenyl)-2-(4-sulphophenyl)-2H-tetrazolium] reduction assay (Promega, Madison, Wisc., USA). Briefly, 60,000 cells/well were grown in flat 96 -well plates for $4 \mathrm{~h}$. Compounds were added to the cells and incubated at $37^{\circ} \mathrm{C}$. Cell viability was measured after $24 \mathrm{~h}$ by adding MTS Reagent (10\%). After $2 \mathrm{~h}$, optical density (OD) was measured at $490 \mathrm{~nm}$ with $630 \mathrm{~nm}$ as the reference wavelength using a spectrophotometer.
Table 2. In vitro activity against a set of 20 clinical isolates of MRSA (MICs in $\mathrm{mg} / \mathrm{l}$ )

\begin{tabular}{llcl}
\hline Compound & MIC 50\% & MIC 90\% & Range \\
\hline NCD9 & 0.25 & 2 & $0.125-2$ \\
NCD10 & 0.25 & 0.5 & $0.125-0.5$ \\
Ciprofloxacin & 32 & $>32$ & 0.06 to $>32$ \\
Chlorhexidine & 0.125 & 0.5 & $0.06-0.5$ \\
\hline
\end{tabular}

\section{Results}

Ten nostocarboline dimers (fig. 1), synthesized with the aim of evaluating linker size and antibacterial properties, were tested against a panel of bacterial and yeast strains (table 1). Monomeric nostocarboline, the biscationic antiseptic chlorhexidine and the fluoroquinolone antibiotic ciprofloxacin were used as comparators. While the nostocarboline monomer was not active at the tested concentrations, all dimers showed antimicrobial activity against at least one strain. Potency and antimicrobial spectrum varied with length and structure of the linker used to connect the monomers. Activities of dimers NCD1-NCD8, with linkers of equal or shorter than eight carbon units, were relatively weak. In contrast, NCD9 and NCD10, with linear C10 and C12 linkers, respectively, showed potent activity with MICs of less than $1 \mathrm{mg} / \mathrm{l}$ for many strains. Against Gram-positive bacteria (S. aureus, Enterococcus faecium, Streptococcus pneumoniae), these compounds were as potent as chlorhexidine, while against Gram-negative bacteria (Haemophilus influenzae, A. baumannii, E. coli, P. aeruginosa), they were less active. Potency increased again significantly in a permeable and efflux-deficient $E$. coli mutant (Ec2085). Interestingly, only NCD10 with the longest linker showed potent activity against the yeast strains Saccharomyces cerevisiae and Candida albicans.

The two most active dimers, NCD9 and NCD10, were further challenged against a collection of 20 recent clinical isolates of MRSA, most of which were resistant to fluoroquinolones (table 2). All strains were susceptible to NCD9 and NCD10 with MICs in the range of 0.125$2 \mathrm{mg} / \mathrm{l}$ and $0.125-0.5 \mathrm{mg} / \mathrm{l}$, respectively, similar in potency to chlorhexidine.

Time-dependent antibacterial killing was investigated in an MRSA strain (table 3). At a concentration of $16 \times$ the MIC, both NCD9 and NCD10 showed a rapid reduction of viable bacteria ( $>99 \%$ within $4 \mathrm{~h}$ ), while at $4 \times$ the MIC, the effect was only static. A similar concentration- 
Fig. 1. Chemical structures of nostocarboline, nostocarboline homodimers and chlorhexidine (CHL).

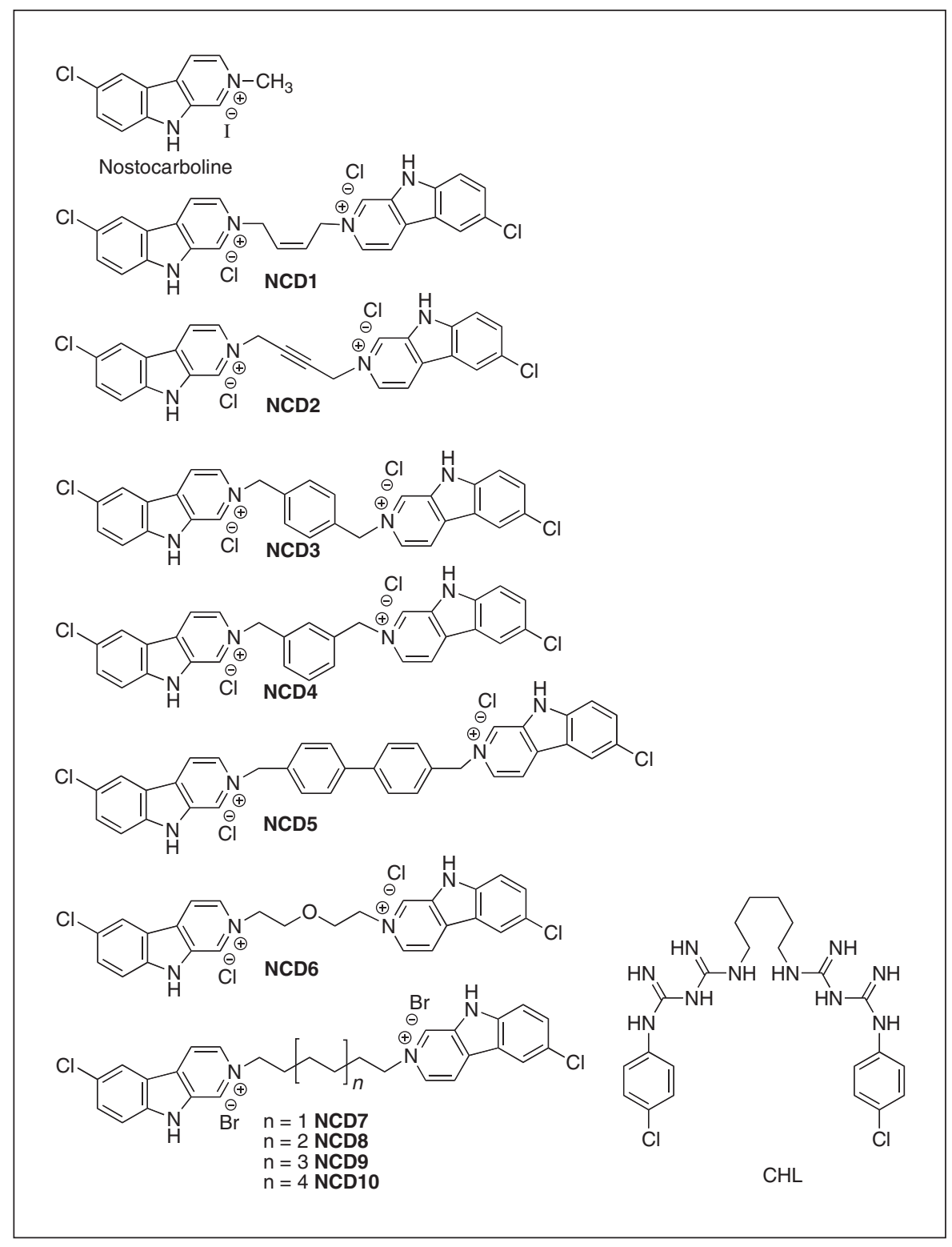

dependent killing effect was also observed with chlorhexidine.

Although the mode of action of chlorhexidine and other polycationic antiseptics is not clear in all details, disruption of the plasma membrane seems to be an important factor for their bactericidal effect $[18,19]$. Bacterial membrane damage exhibited by nostocarboline dimers was examined by measuring the leakage of cellular ATP (table 3). Short-term exposure $(20 \mathrm{~min})$ of cultures of $S$. aureus to NCD9 and NCD10 at $16 \times$ the MIC led to strong ATP release (30-40\% of SDS-treated positive control), while at $4 \times$ the MIC, only a minor effect was ob- served compared to the untreated control. Chlorhexidine also exhibited a concentration-dependent ATP leakage, while ciprofloxacin, as expected, had no short-term effect on membrane integrity. The observed concentrationdependent ATP leakage exhibited by NCD9 and NCD10 goes parallel with the rapid concentration-dependent bactericidal effect and suggests that interference with plasma membrane integrity is at least one of the reasons for their bactericidal effect.

To test specificity for bacterial cells, the two most active nostocarboline dimers were tested for cytotoxicity in $\mathrm{CHO}$ cells (table 4). Cytotoxic activities of the nostocar- 

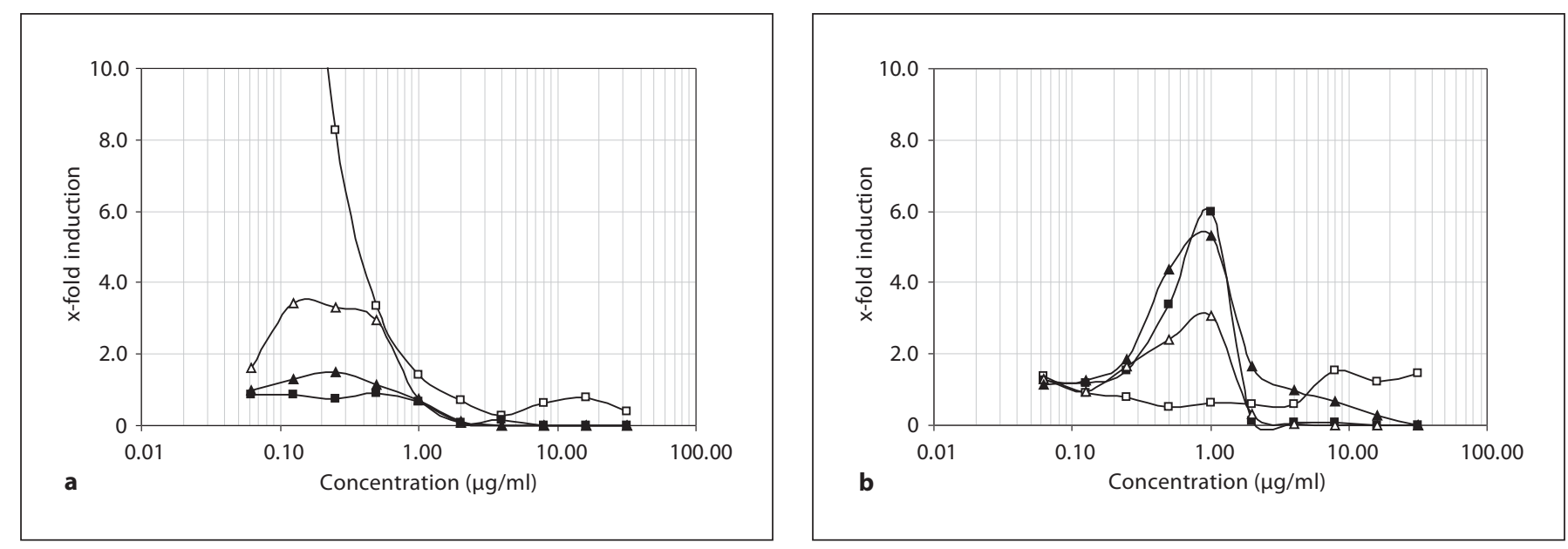

Fig. 2. Induction of stress response reporters by antibacterial compounds ciprofloxacin (CIP; open squares), chlorhexidine (CHL; filled squares), NCD9 (open triangles) and NCD10 (filled triangles). SOS induction measured in Ec2376 [ $\Delta$ tolC $\left.\Delta r f a C \Delta l a c Z::\left(s u l A^{\prime}-' l u x C D A B E\right)\right] 2 \mathrm{~h}$ after addition to compound (a) and induction of cell-envelope stress response in Ec2297 [ $\Delta$ tolC $\left.\Delta l a c Z::\left(\operatorname{deg} P^{\prime}-{ }^{\prime} l u x C D A B E-\right)\right]$ (b). Three independent experiments were performed and representative data is shown.

Table 3. Bactericidal effect and release of cellular ATP in S. aureus

\begin{tabular}{|c|c|c|c|}
\hline \multirow[t]{2}{*}{ Compound } & \multicolumn{2}{|c|}{ log CFU change } & \multirow{2}{*}{$\begin{array}{l}\text { Release of cellular } \\
\text { ATP, \% of SDS- } \\
\text { lysed control }\end{array}$} \\
\hline & $1 \mathrm{~h}$ & $4 \mathrm{~h}$ & \\
\hline Untreated control & +0.84 & +2.07 & 0.5 \\
\hline NCD9 $(4 \times$ MIC $)$ & -0.11 & -0.15 & 4.4 \\
\hline NCD9 $(16 \times$ MIC $)$ & -1.31 & -2.61 & 41.1 \\
\hline NCD10 $(4 \times$ MIC $)$ & +0.25 & +0.01 & 2.6 \\
\hline NCD10 $(16 \times$ MIC $)$ & -0.96 & -3.28 & 33.2 \\
\hline Ciprofloxacin $(4 \times$ MIC) & -0.7 & -2.8 & 0.8 \\
\hline Ciprofloxacin $(16 \times$ MIC $)$ & -1.5 & -2.6 & 0.7 \\
\hline Chlorhexidine $(4 \times$ MIC) & -0.14 & -1.26 & 2.3 \\
\hline Chlorhexidine $(16 \times$ MIC $)$ & -0.9 & -4.1 & 9.8 \\
\hline
\end{tabular}

ATP in supernatant was measured by luminescence assay. $0.1 \%$ SDS was used as a membranolytic control and relative light units obtained were arbitrarily set as $100 \%$.

boline dimers were relatively weak compared to the MIC against $S$. aureus strains. NCD9 was significantly less toxic than NCD10 and both dimers were clearly less toxic than chlorhexidine. This is in agreement with the finding that NCD10 (and chlorhexidine) is active against yeast cells while NCD9 is not. This observation suggests
Table 4. Cytotoxicities of selected dimers of nostocarboline compared to chlorhexidine

\begin{tabular}{lcc}
\hline Compound & \multicolumn{2}{c}{ Cytotoxicity in $\mathrm{CHO}$ cells $\left(\mathrm{IC}_{50}\right.$ in $\left.\mathrm{mg} / \mathrm{l}\right)$} \\
\cline { 2 - 3 } & $24 \mathrm{~h}$ & $48 \mathrm{~h}$ \\
\hline NCD9 & $>50$ & 23.8 \\
NCD10 & 25.7 & 14.2 \\
Chlorhexidine & 13.9 & 1.7 \\
Ciprofloxacin & $>50$ & $>50$ \\
\hline
\end{tabular}

Data are averages of three determinations.

that NCD9 with a C10 linker has better selectivity for prokaryotic cells than NCD10 with a C12 spacer.

The mechanism of action of NCD9 and NCD10 was also tested in E. coli reporter strains lacking the multidrug resistance TolC pump. The sulA fusion strain Ec2376 was used to detect induction of the SOS response following DNA damage as described previously [20]. Additionally, the DegP reporter strain Ec2297 was used to monitor the cell envelope stress response [21, 22]. Only NCD9 induces an SOS response, albeit at a much weaker degree than ciprofloxacin (fig. 2a), whereas NCD10 (and, more weakly, NCD9) elicited cell envelope stress in a similar fashion as chlorhexidine (fig. 2b). 


\section{Conclusion}

Cationic antimicrobials are widely used as disinfectants and preservatives and in topical antimicrobial products [18]. On the other hand, cationic antimicrobial peptide antibiotics, such as the polymyxins, have also proved to be useful for systemic antibacterial treatment and colistin (polymyxin E) is considered as a last resort drug in the treatment of infections involving some multidrug-resistant bacteria [23]. While the mechanism of antibacterial action is not elucidated in all details, early damage to the cytoplasmic membrane leading to lethal effects is considered to be the common theme for this class of compounds [19]. One or more strong positive charges, together with a hydrophobic region, are the basic structural requirements for membrane interaction. However, there is still controversy whether other interactions including intracellular targets are involved, leading to the final lethal effect [24]. Furthermore, the mechanism of action may vary considerably within this class of compounds $[25,26]$. The low propensity for resistance development due to multiple target sites, the rapid bactericidal effect and the potentially broad antimicrobial spectrum are properties that make this antimicrobial class attractive for future treatment of bacterial infections, including bacteria resistant to all currently available antibiotics $[26,27]$. However, there are several problems associated with the development of such compounds for (systemic) therapeutic use, e.g. the lack of specificity and consequently potentially toxic side effects, poor bioavailability and stability, and lack of systemic in vivo activity [26-28]. In this report, we show that some homodimeric compounds (biscarbolines) created from the natural alkaloid nostocarboline display potent antibacterial activity. Structurally, the nostocarboline dimers can be compared to bisbiguanide antiseptics, such as chlorhexidine, i.e. bipolar positive charges separated by a hydrophobic spacer region. Antimicrobial potencies and spectra of the dimers varied with length of the linker used to connect the two carbolinium moieties: the derivatives with linear $\mathrm{C} 10$ - and C12-linkers were highly potent against Gram-positive bacteria while compounds with a shorter linker had only modest activities and the nostocarboline monomer was essentially inactive. The length of the hydrophobic spacer is also important for antibacterial activity of the bisguanides and a six carbon bridge seems to be ideal for activity [19]. In contrast, quaternary ammonium antiseptics are monocationic with a long hydrophobic tail. However, these compounds show dimerization in solution and, consequently, the dimeric structure may be responsible for antibacterial activity.
The two most active nostocarboline dimers, NCD9 and NCD10, showed potent Gram-positive activity especially against $S$. aureus, including multi-resistant clinical MRSA isolates. On the other hand, activities against Gram-negative bacteria were relatively weak, in particular against $P$. aeruginosa. Poor penetration through the outer membrane and/or intensive efflux are possible reasons for that finding. This notion is underscored by the strongly increased activities in E. coli efflux k.o. and outer membrane permeability mutants. Interestingly, NCD9 with a C10 linker seems to act in a more specific way than NCD10 with a $\mathrm{C} 12$ spacer as indicated by lower cytotoxicity and absence of activity against yeast strains. It is tempting to speculate that selectivity to bacteria versus eukaryotic cells may depend on the length of the linker. Preliminary investigations addressing the mode of action in bacteria suggest that the nostocarboline dimers act on Gram-positive bacteria in a similar way as chlorhexidine, i.e. by concentration-dependent membrane damage leading to leakage of intracellular material followed by rapid cell death. In reporter strains of efflux-deficient $E$. coli, we found induction of DegP response pathway, consistent with the data above suggesting damage to the cell envelope including the proton motive force. It has been shown that the DegP expression is regulated by the $\mathrm{CpxR}$ regulator and depends on $\sigma \mathrm{E}$, which in turn can sense misfolded proteins in extracellular compartments or defects in the biogenesis of outer membrane proteins, respectively [21]. It has also been shown that membrane-active compounds that abolish the proton motive force induced the DegP-dependent stress response pathway [22]. Therefore, it was quite intriguing to find that NCD9, but not NCD10, also induced the SOS response, as this would suggest that the compound could interact with a cytoplasmic target as well.

Nostocarboline and its dimers have also shown potent anti-plasmodial activity [10], but the structure activity relationship is different from the one observed in this study. In particular, nostocarboline was determined to possess submicromolar activity against $P$. falciparum. The dimers showed consistent activity below $100 \mathrm{nM}$ for dimers NCD6-NCD10 against this parasite. This different structure/activity relationship suggests that different modes of action are involved.

The results presented in this report indicate the potential of this new class of cationic antimicrobial compounds for the design of potent and selective antibacterials with low cytotoxicity. Further studies should involve the evaluation of antibacterial activity and toxicity in in vivo models and the design of new compounds with improved Gram-negative activity while optimizing selectivity. 


\section{Acknowledgements}

Part of this work was funded by Actelion Pharmaceuticals Ltd. K.G. is a European Young Investigator and thanks the Swiss National Science Foundation for their support (PE002-117136/1). Funding by ETH Zürich is gratefully acknowledged (TH Gesuch 13/04-3).

\section{Disclosure Statement}

H.H.L., D.R., P.P., M.G., A.K., D.S. and S.S. are employees of Actelion Pharmaceuticals Ltd., Allschwil, Switzerland and own/ have owned stocks or shares of Actelion.

\section{References}

$\checkmark 1$ Boucher HW, Talbot GH, Bradley JS, Edwards JE, Gilbert D, Rice LB, Scheid M, Spellberg B, Bartlett J: Bad bugs, no drugs: no ESKAPE! An update from the Infectious Diseases Society of America. Clin Infect Dis 2009;48:1-12.

-2 Spellberg B, Guidos R, Gilbert D, Bradley J, Boucher HW, Scheld WM, Bartlett JG, Edwards J Jr, Infectious Diseases Society of America: The epidemic of antibiotic-resistant infections: a call to action for the medical community from the Infectious Diseases Society of America. Clin Infect Dis 2008;46: 155-164.

3 Al-Tawfik JA, Anani AA: Antimicrobial susceptibility pattern of bacterial pathogens causing urinary tract infections in a Saudi Arabian hospital. Chemotherapy 2009;55: 127-131.

4 Lange RP, Locher HH, Wyss PC, Then RL: The targets of currently used antibacterial agents: lessons for drug discovery. Curr Pharm Des 2007;13:3140-3154.

5 Newman DJ, Cragg GM: Natural products as sources of new drugs over the last 25 years. Nat Prod 2007;70:461-477.

-6 Gademann K, Portmann C: Secondary metabolites from cyanobacteria: complex structures and powerful bioactivities. Curr Org Chem 2008;12:326-341.

7 Blom JF, Brütsch T, Barbaras D, Bethuel Y, Locher HH, Hubschwerlen C, Gademann K: Potent algicides based on the cyanobacterial alkaloid nostocarboline. Org Lett 2006;8: 737-740.

8 Portmann C, Blom JF, Gademann K, Jüttner F: Aerucyclamides A and B: isolation and synthesis of toxic ribosomal heterocyclic peptides from the cyanobacterium Microcystis aeruginosa PCC 7806. J Nat Prod 2008;71: 1193-1196.

-9 Becher PG, Beuchat J, Gademann K, Jüttner F: Nostocarboline: isolation and synthesis of a new cholinesterase inhibitor from Nostoc 78-12A. J Nat Prod 2005;68:1793-1795.
10 Barbaras D, Kaiser M, Brun R, Gademann K: Potent and selective antiplasmodial activity of the cyanobacterial alkaloid nostocarboline and its dimers. Bioorg Med Chem Lett 2008;18:4413-4415

11 Bonazzi S, Barabaras D, Patiny L, Scopelliti R, Schneider P, Cole ST, Kaiser M, Brun R, Gademann K: Antimalarial and antitubercular nostocarboline and eudistomin derivatives: synthesis, in vitro and in vivo biological evaluation. Bioorg Med Chem 2010;18 1464-1476.

12 Winson MK, Swift S, Hill PJ, Sims CM, Griesmayr G, Bycroft BW, Williams P, Stewart GS: Engineering the luxCDABE genes from Photorhabdus luminescens to provide a bioluminescent reporter for constitutive and promoter probe plasmids and mini-Tn 5 constructs. FEMS Microbiol Lett 1998;163:193202.

13 Datsenko KA, Wanner BL: One-step inactivation of chromosomal genes in Escherichia coli K-12 using PCR products. Proc Natl Acad Sci USA 2000;97:6640-6645.

14 Gérard F, Pradel N, Wu LF: Bactericidal activity of colicin $\mathrm{V}$ is mediated by an inner membrane protein, $\mathrm{SdaC}$, of Escherichia coli. J. Bacteriol 2005;187:1945-1950.

15 Miller JH: A Short Course in Bacterial Genetics: A Laboratory Manual and Handbook for Escherichia coli and Related Bacteria. Cold Spring Harbor, Cold Spring Harbor Laboratory, 1992.

16 Clinical and Laboratory Standards Institute: Methods for Dilution Antimicrobial Susceptibility Tests for Bacteria That Grow Aerobically, ed.7.: Approved Standard M7-A7. Wayne, CLSI, 2006.

$>17$ Higgins DL, Chang R, Debabov DV, Leung J, Wu T, Krause KM, Sandvik E, Hubbard JM, Kaniga K, Schmidt DE Jr, Gao Q, Cass RT, Karr DE, Benton BM, Humphrey PP: Telavancin, a multifunctional lipoglycopeptide, disrupts both cell wall synthesis and cell membrane integrity in methicillin-resistant Staphylococcus aureus. Antimicrob Agents Chemother 2005;49:1127-1134.
18 McDonnell G, Russell AD: Antiseptics and disinfectants: activity, action, and resistance. Clin Microbiol Rev 1999;12:147-179.

19 Gilbert P, Moore LE: Cationic antiseptics: diversity of action under a common epithet. J Appl Microbiol 2005;99:703-715.

20 Quillardet P, de Bellecombe C, Hofnung M: The SOS chromotest, a calorimetric assay for genotoxins: validation study with 83 compounds. Mut Res 1985;147:79-95.

21 Raivio T: Envelope stress response and Gram-negative pathogenesis. Mol Microbiol 2005;56:1119-1128.

22 Andersen CL, Holland IB, Jacq A: Verapamil, a Ca ${ }^{2+}$ channel inhibitor acts as a local anesthetic and induces the sigma $\mathrm{E}$ dependent extra-cytoplasmic stress response in $E$. coli. Biochim Biophys Acta 2006; 1758:15871595.

23 Zapantis A, Lopez M, Hoffman E, et al: The use of colistin in multidrug-resistant infections. Hosp Pharm 2007;42:1127-1138.

24 Friedrich CL, Moyles D, Beveridge T, Hancock REW: Antibacterial action of structurally diverse cationic peptides on Gram-positive bacteria. Antimicrob Agents Chemother 2000;44:2086-2092.

25 Jenssen H, Hamill P, Hancock RE: Peptide antimicrobial agents. Clin Microbiol Rev 2006;19:491-511.

$26 \mathrm{Wu}$ MH, Maier E, Benz R; Hancock RE: Mechanism of interaction of different classes of cationic antimicrobial peptides with planar bilayers and with the cytoplasmic membrane of Escherichia coli. Biochemistry 1999; 38:7235-7242.

27 van't Hof W, Veerman ECI, Helmerhorst EJ, Amerongen AV: Antimicrobial peptides: properties and applicability. Biol Chem 2001; 382:597-619.

28 Ryge TS, Frimodt-Moller N, Hansen PR: Antimicrobial activities of twenty lysinepeptoid hybrids against clincaly relevant bacteria and fungi. Chemotherapy 2008;54: 152-156. 